\title{
On-machine form measurement of high precision ceramics parts by using a laser displacement sensor
}

\author{
So ITO*, Daiki MATSUURA*, Takayuki MEGURO*, Shigeaki GOTO*, Yuki SHIMIZU*, Wei GAO*, \\ Shigeru ADACHI** and Kyohei OMIYA** \\ *Department of Nanomechanics, Tohoku University \\ 6-6-01 Aramaki Aza Aoba, Aoba-ku Sendai, Miyagi, 980-8579, Japan \\ E-mail: so.ito@nano.mech.tohoku.ac.jp \\ **JAPAN FINE CERAMICS Co., LTD., \\ Akedori 3-10, Izumi-ku, Sendai, Miyagi, 981-3206, Japan
}

Received 27 February 2014

\begin{abstract}
Ceramics materials have excellent mechanical characteristics such as high-stiffness, low-thermal expansion, wear resistance and light weight. Owing to those characteristics, the ceramics parts have been used for the sliding part of the precision positioning stages. However, it is difficult for the ceramics workpieces to achieve the desired form accuracy in once machining process because of the brittleness of the ceramics materials. The compensation of the form error is processed by the manual procedures in general, so it requires a skill of the operator and a great deal of time. In order to realize the automation of the error compensation, it is necessary to measure the workpiece form on the machine tool. In this study, an on-machine form measurement system using a laser displacement sensor having nanometric accuracy is introduced for the surface form measurement of the precision ceramics workpieces. The laser displacement sensor is mounted on the tool post of the ultra-precision polishing machine to realize the on-machine measurement of the ceramics workpieces. By using two horizontal linear slides of the machine tool, the laser displacement sensor can scan the surface of the workpiece with sub-micrometric motion accuracy. Consequently, three-dimensional continuous surface form of the precisely machined ceramics workpiece can be obtained in non-contact condition. In order to evaluate the measurement accuracy of the constructed system, the experiments to investigate stability and motion accuracy were conducted, and then the form measurement of the precision ceramics parts is carried out by using the proposed measurement system.
\end{abstract}

Key words: Ceramics, On-machine measurement, Non-contact measurement, Laser displacement sensor, Ultra-precision machining tool, Ultra-precision grinding

\section{Introduction}

Ultra-precision positioning stages are utilized in various instruments such as ultra-precision machine tools, semiconductor manufacturing equipments, optical apparatus, and precision measurement instruments (Nakamoto, 2006). With the high density and miniaturization of the devices, the precision positioning stages have been required both of nanometric positioning accuracy and travel range of several hundred millimeters. The positioning accuracy and motion accuracy of the positioning stages are relied on the form accuracy of the sliding surface. In general, the sliding part of the positioning stage is made of the metallic material. However, the form accuracy of the metallic sliding part is not enough for the ultra-precision positioning with nanometric accuracy because of thermal expansion, wear of the sliding surface and its own weight (Ostuka, 2009). Therefore, in the recent years, the sliding parts made of the ceramics materials are employed for the ultra-precision positioning stages. Ceramics materials have the excellent mechanical characteristics such as high-stiffness, low thermal expansion, wear resistance, and light weight (Sugawara, 2013).

In order to realize the nanometric positioning accuracy, the form accuracy of sub-micrometer order is required for the sliding surface of the ceramics parts. The ceramics parts are usually fabricated by various processes such as cutting 
(Moriwaki et al., 1992), polishing (Suzuki et al., 2010) and laser process (Kuwata et al., 1993). With regard to the machining of the precision ceramics parts, the polishing process has been employed due to the brittleness of ceramics materials and manufacturing cost (Sato and Komatsuzaki, 1992). However, it is difficult for the ceramics parts to achieve sub-micrometric accuracy by single polishing process because the grinding tool will be worn during the polishing due to the hardness of the ceramics material (Goto, et al., 2012, Shimizu et al., 2013). Since the form of the machined surface is transferred of the tool shape by the tool motion, the wear of the grinding tool induces the form error with respect to the design shape. In order to fabricate the precision ceramics parts with sub-micrometric form accuracy, the error compensation has been carried out by the manual procedures. In general, the finishing process of the ceramics workpiece is implemented by the manual procedures such as a scrapping and lapping. However, those manual procedures require the skill of the operator. Furthermore, since the form measurement and the finishing process are repeated alternately to correct the form error of the ceramics workpieces, the error compensation processing by the manual procedures take a lot of times.

With regard to another effort to achieve sub-micrometric form accuracy of the precision ceramics parts, an automatic compensation machining is one of useful method for improvement of the machining accuracy and efficiency of manufacturing. This compensation process is conducted based on the form measurement after the first machining process. Generally, the machined ceramics workpiece must be detached from the table of the polishing machine for the measurement. Then, the ceramics workpiece is fixed on the measurement instrument such as a coordinate measurement machine and surface roughness tester to evaluate the dimensions and surface roughness of the ceramics workpiece. However, after the ceramics workpiece is removed from the polishing machine for the measurement, it is difficult for the workpiece to be reattached to the same position on the polishing machine with sub-micrometric accuracy. Therefore, in order to avoid the misalignment due to the attachment and detachment of the workpiece, the on-machine measurement systems have been required (Mou and Richard, 1992, Onaka and Nakagawa, 2005, Rahman et al., 2008). With the increase in the size of the sliding parts made of the ceramics materials, the on-machine measurement system is required not only sub-micrometric measurement accuracy but also the measurement range of several hundred millimeters. With regard to the on-machine measurement of the ceramics workpiece, a touch probe has been used (Rahman et al., 2008, Ueno, 2009). However, the realized range and accuracy of such a system is not satisfied for the measurement of the measurement of the sliding part. In addition, since the surface of the ceramics parts was measured by the touch probe, it was difficult to obtain continuous surface profile.

In this study, a surface form measurement system with a laser displacement sensor is developed for the measurement of the precision machined ceramics workpiece. The measurement system is consisted on the tool post on the precision polishing machine. Since use of the laser displacement sensor allow non-contact measurement of the workpiece surface, it achieve shorten of the measurement time. The design of the constructed on-machine measurement system is described. Stability and motion error of the measurement system are investigated to evaluate the measurement accuracy. The purpose of this study is to realize the on-machine measurement with sub-micrometric accuracy.

Table 1 Specification of the linear slides stage of the ultra-precision polishing machine

\begin{tabular}{c|c|c}
\hline \hline \multirow{4}{*}{ Range } & $X$ & $300 \mathrm{~mm}$ \\
\cline { 2 - 3 } & $Y$ & $25 \mathrm{~mm}$ \\
\cline { 2 - 3 } & $Z$ & $150 \mathrm{~mm}$ \\
\hline \multirow{4}{*}{ Straightness } & $Y$ & $0.2 \mu \mathrm{m} / 300 \mathrm{~mm}$ \\
\cline { 2 - 3 } & $Y$ & $1.5 \mu \mathrm{m} / 25 \mathrm{~mm}$ \\
\cline { 2 - 3 } & $Z$ & $0.1 \mu \mathrm{m} / 150 \mathrm{~mm}$ \\
\hline \hline
\end{tabular}

Table 2 Specification of triangulation method laser displacement sensor

\begin{tabular}{c|c}
\hline \hline Range & $\pm 0.5 \mathrm{~mm}$ \\
\hline Wavelength & $655 \mathrm{~nm}$ \\
\hline Accuracy & $0.005 \mu \mathrm{m}$ \\
\hline Linearity & $\pm 0.05 \%$ of F.S. \\
\hline \hline
\end{tabular}




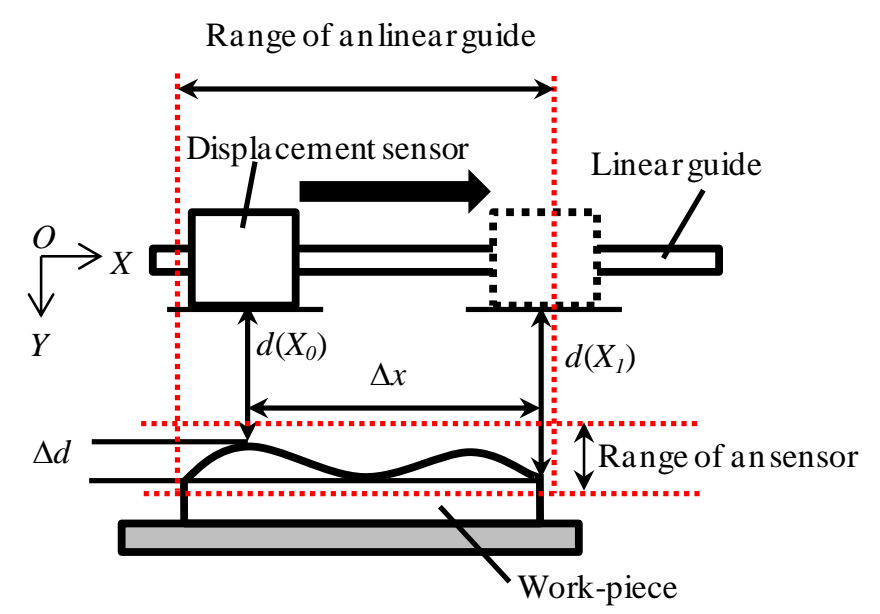

Fig. 1 Schematic diagram of the constructed on-machine measurement system

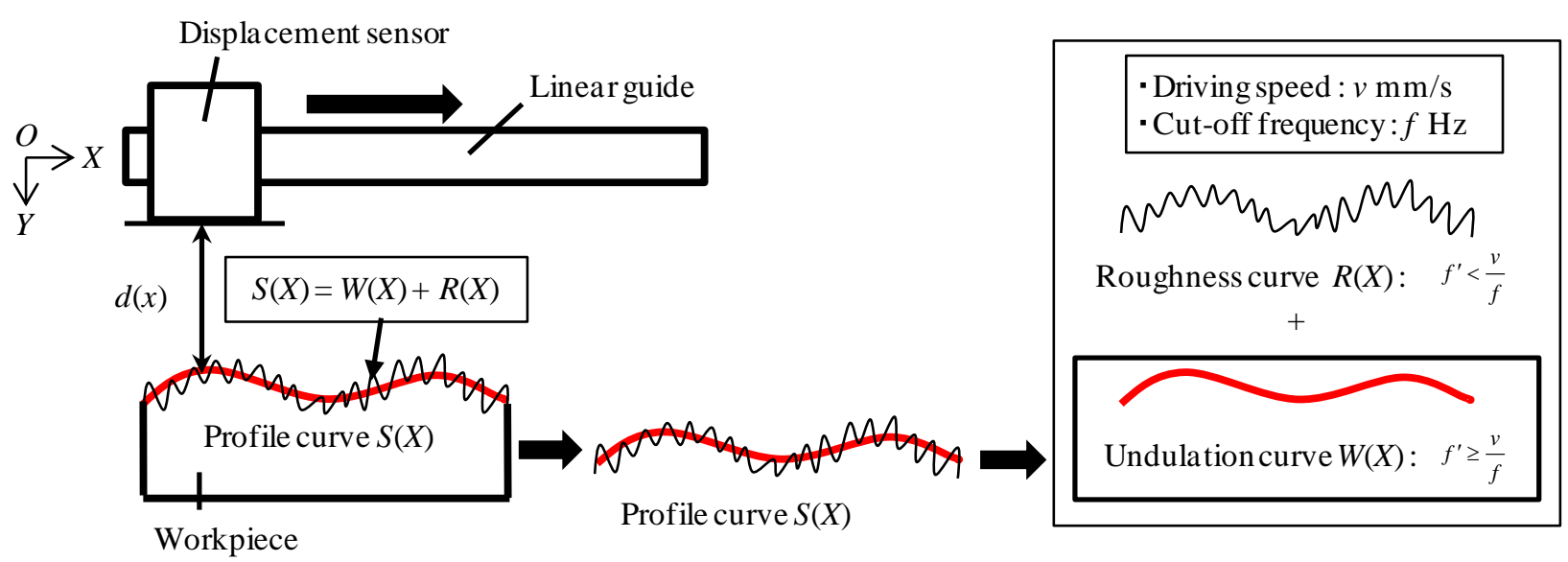

Fig. 2 Principle of the undulation curve measurement with a laser displacement sensor

\section{On-machine measurement system}

\subsection{Principle of form measurement}

An on-measurement system is constructed by using a laser displacement sensor. A schematic diagram of the constructed system is shown in Fig. 1. The laser displacement sensor is mounted on the tool post of the ultra-precision polishing machine (ULG-100D, Toshiba Machine). A ceramics workpiece is fixed on the table of the polishing machine. Table 1 shows a specification of the linear slides of the ultra-precision polishing machine used in this study. As shown in Table 1, the linear slides of the polishing machine have working range of several hundred millimeters in $X$ and $Z$ directions. In addition, sub-micrometric straightness can also be realized in $X$ and $Z$ directions. Consequently, the linear stages to be used in this study allow the straightness of the sub-micrometer scale in the scanning range of the millimeter-order. The distance $d(x)$ between the sensor and the surface of the ceramics workpiece can be measured by the displacement sensor. Here, $x$ is defined as the position of the displacement sensor in $X$ direction. Since the sensor displacement in $X$ direction $\Delta x$ can be detected by a linear encoder which is installed the linear slide of the polishing machine, the resolution of $\Delta x$ is relied on the feed rate of the linear slide and the sampling frequency of the displacement sensor. On the other hand, $\Delta d$, which indicates the peak-to-valley value of the surface form on the measuring profile between two sampling points $X_{i}$ and $\underline{X}_{i+1}$, can be obtained by the output of the displacement sensor. Therefore, $\Delta d$ can be determined by the output of the displacement on each sampling position.

Figure 2 shows the principle of the undulation curve measurement. The measuring curve of the surface form profile is composed of two curves, which are roughness curve and undulation curves. Those two curves are possible to be 


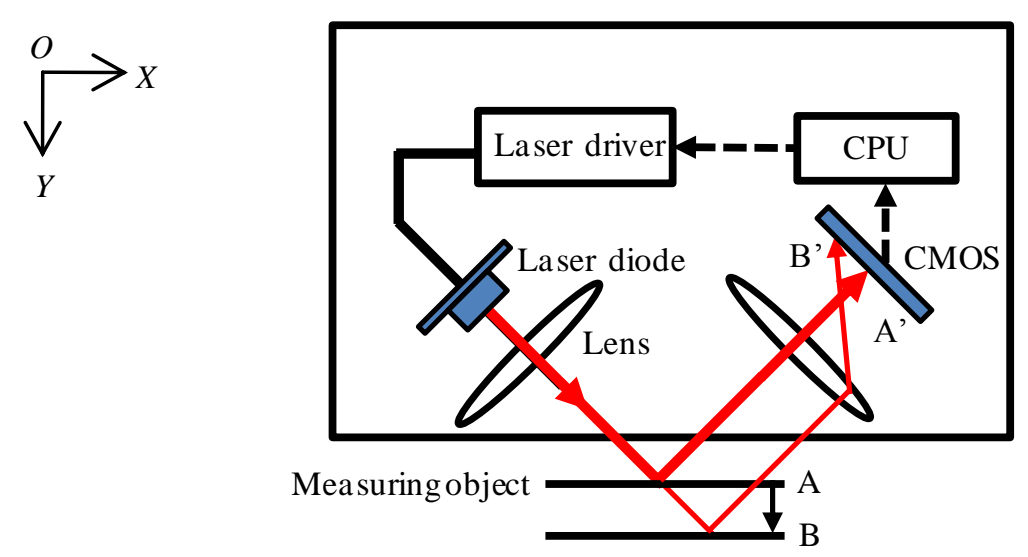

Fig. 3 Schematic diagram of a triangulation method laser displacement sensor

distinguished by the difference of the spatial frequency. The roughness curve is constituted by a high spatial frequency, while, the undulation curve is constituted by a low frequency. Positioning accuracy and motion accuracy of the positioning stage is influenced by the undulation of the sliding part. According to ISO 4287, the cutoff value of the undulation curve is more than $0.8 \mathrm{~mm}$ of the wavelength. The wave length is defined as an inverse of the spatial frequency. In order to obtain the undulation curve, it is necessary to obtain the surface profile with more than $0.8 \mathrm{~mm}$ of the cutoff value. While the stage of the polishing machine is scanned in $X$ direction at feed rate $v \mathrm{~mm} / \mathrm{s}$, the displacement sensor can measure the distance $d(x)$ continuously. High-frequency components of the sensor output can be removed by utilizing a digital low-pass filter of cutoff frequency $f_{C O F}$. Therefore, the spatial frequency component of the surface form profile that is obtained by the laser displacement sensor can be expressed as follows.

$$
f^{\prime}=\frac{v}{f_{C O F}}
$$

where, $f$ ' is the cutoff spatial frequency of the measurement system. Since the displacement sensor can scan at a constant feed rate along the $X$ direction with the linear slide of polishing machine, the accuracy of the form profile measurement depends on the linearity of the positioning stage. With regard to the accuracy of the form profile measurement in this study, the straightness of the linear stage and the accuracy of the displacement sensor are important factor. Therefore, it is necessary to evaluate those parameters of the measurement system.

Measurement accuracy of the displacement sensor also has an effect on the measurement accuracy of the form profile. As for the displacement sensor, there are two types of displacement sensor, which are represented by a contact-type and noncontact-type. However, it is difficult for the contact-type displacement to measure the form profile continuously at high speed because the bandwidth of the sampling frequency. Furthermore, the contact-type sensors have some risks of the damaging on the workpiece. On the other hand, the noncontact-type displacement sensor can prevent those issues. There are several types of the noncontact-type displacement sensors such as a capacitive sensor, an ultrasonic sensor, and eddy-current sensor, and laser displacement sensor. Considering the accuracy of the sensor and the insulating properties of the ceramics materials, the laser displacement sensor is employed in this study. Furthermore, a triangulation method type displacement sensor has been utilized owing to its compact size because the displacement sensor has been required to be enough small to be attached the tool post of the polishing machine. Figure 3 shows a schematic diagram of the triangulation method type laser displacement sensor. The displacement of the measuring surface in $Z$ direction can be detected as the displacement of the reflected light position on the CMOS sensor. However, it is difficult to assure the precise measurement with a laser triangulation displacement sensor because the intensity of the reflected light might be insufficient owing to the low reflectance of the ceramics materials. In order to avoid the decrease of the reflected light power, the output power of the laser probe is controlled to maintain the intensity of the reflected light constant. Table 2 shows a specification of the laser displacement sensor. The accuracy of this sensor is enough for a sub-micrometric form measurement. 


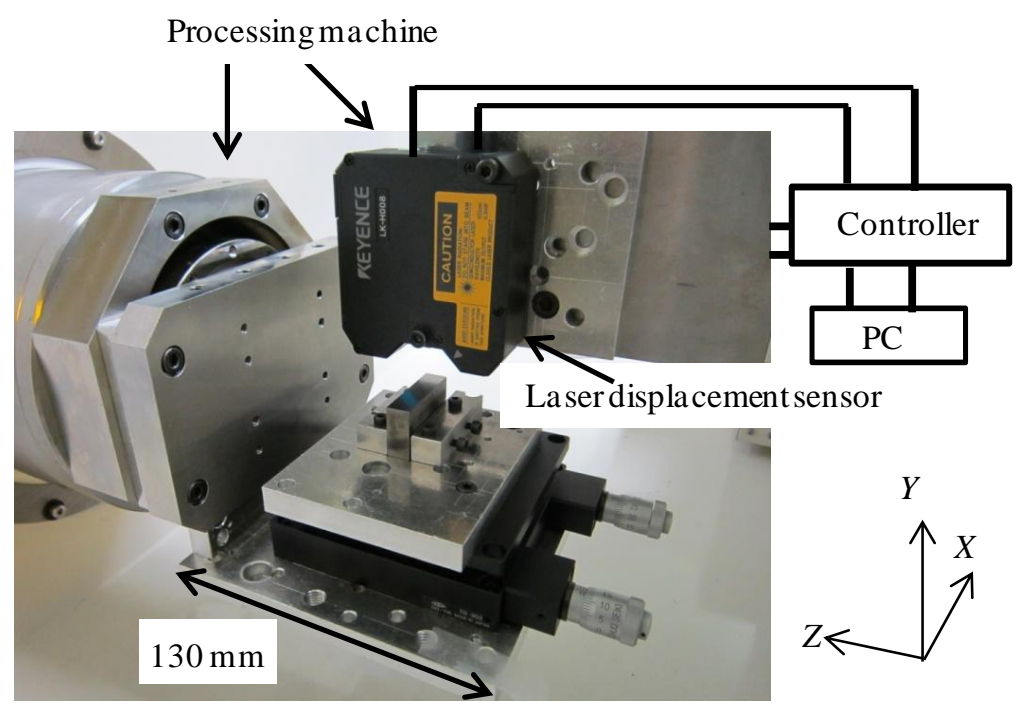

Fig. 4 Experimental configuration of the constructed on-machine measurement system

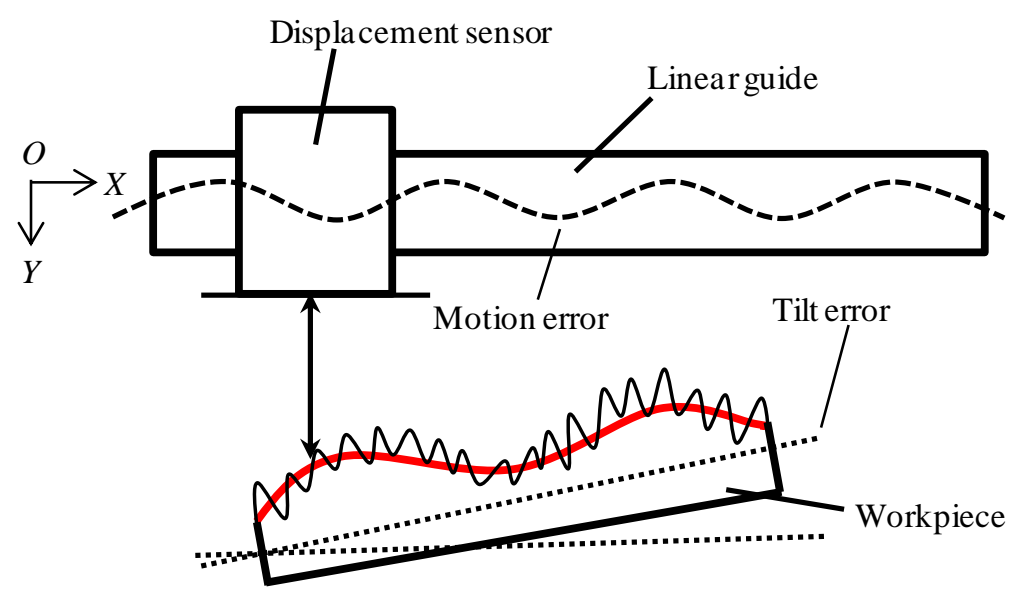

Fig. 5 Schematic of the error factor of the on-machine measurement accuracy

Figure 4 shows the experimental configuration of the constructed form measurement system with laser triangulation displacement sensor. The laser displacement sensor, which is mounted on the tool post of the polishing machine, can scan the surface of the ceramics workpiece by using the two horizontal axes of the polishing machine. Since the linear stages of the ultra-precision positioning machine can realize the precision positioning in three axial directions, it is possible to achieve three dimensional measurement of the ceramics workpiece by utilizing a laser displacement sensor as well as the CMM.

\subsection{Factor of the measurement errors}

In the on-machine measurement of the precision ceramics workpiece, various error factor influence the measurement accuracy. Those error factors can mainly be classified into three types of the errors. Figure 5 shows a schematic of the error factors which influence the measurement accuracy. Firstly, a tilt error between the linear guide and the workpiece is significant factor for the surface form profile measurement. When the ceramics workpiece has been fixed with some inclination, the linear guide of the polishing machine is not parallel to the measuring surface. If the measuring surface becomes outside the measuring range of the displacement sensor due to the inclination of the 


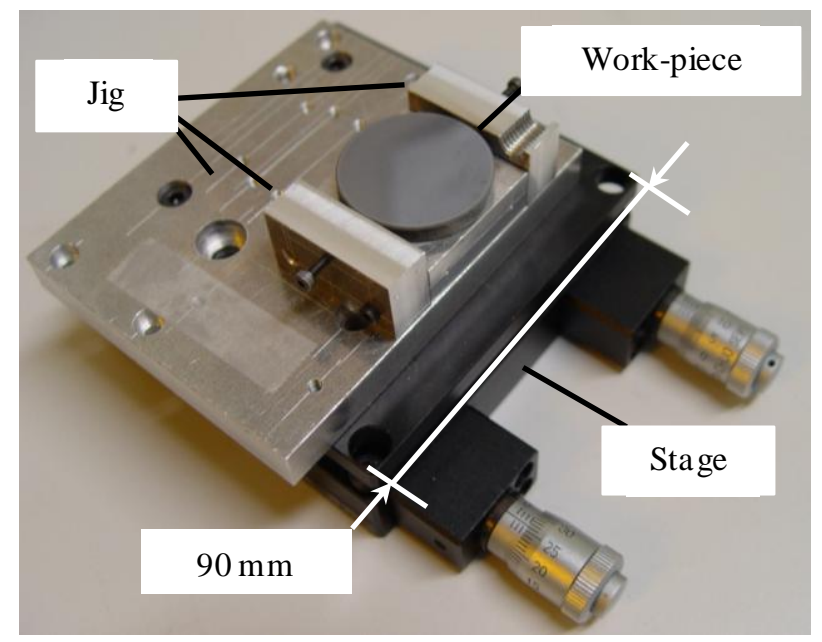

(a) A ceramics workpiece mounted on the tilt stage

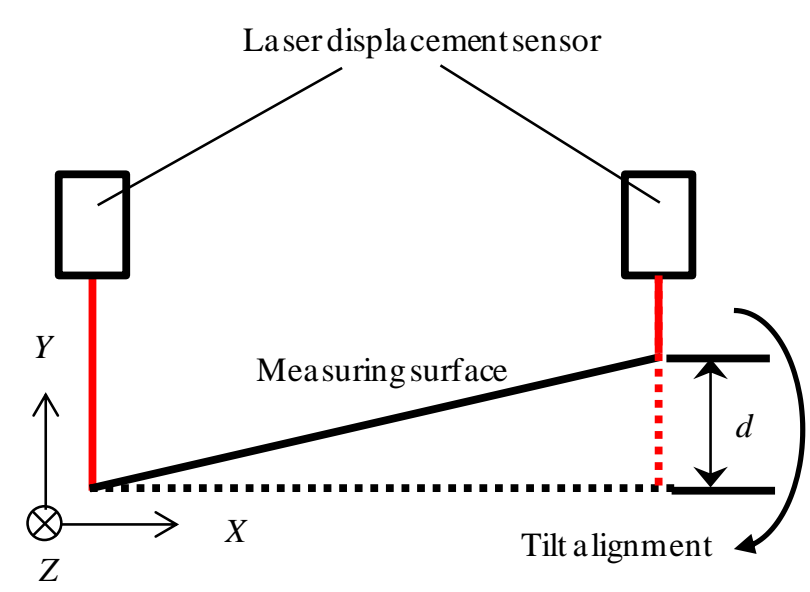

(b) Schematic diagram of the angle compensation method with the tilt of the stage

Fig. 6 Schematic of the inclination compensation by using a tilt stage

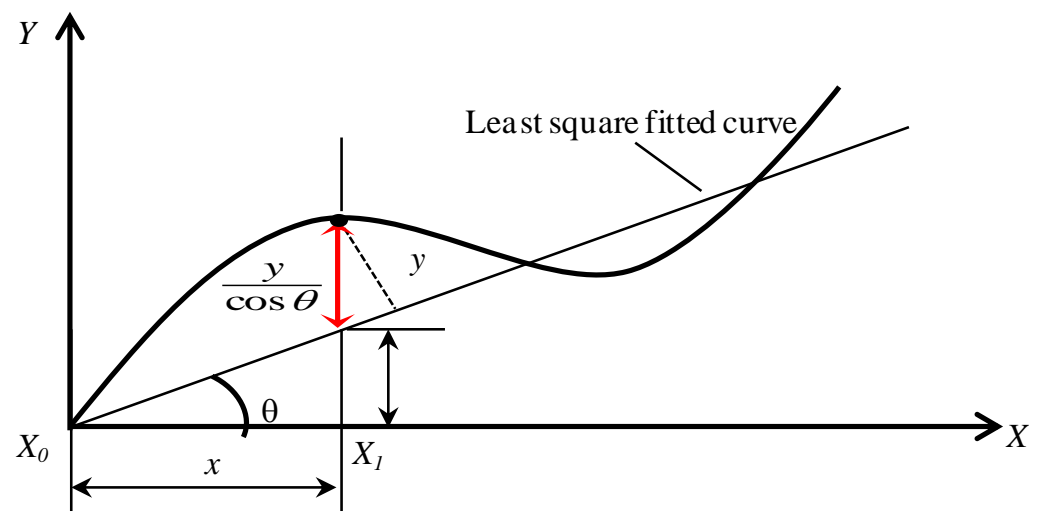

Fig. 7 Schematic of the measurement error caused by the tilt of the workpiece surface

workpiece surface, the form profile measurement cannot be carried out. Furthermore, since the undulation of the workpiece surface is calculated by the peak-to-valley value of the surface form on the measuring profile, the measurement result including the inclination causes the measurement error. Secondly, the stability of the laser displacement sensor has to be estimated because it will affect the repeatability of the form profile measurement. Since the reflectivity of the ceramics materials is lower than that of the metallic material, it should be also evaluated the stability of the output of the laser displacement meter. Finally, the motion error of the linear stage of the machine tool affects the measurement accuracy since the laser displacement sensor, which is used for the measurement of the sensor-to-surface distance, is scanned by utilizing the linear stage of the polishing machine. In this paper, the influences of those error factors are evaluated experimentally.

\section{Evaluation of the error factors}

\subsection{Tilt error}

The tilt error is occurred by the inclination of the measurement surface with respect to the linear guide that the displacement sensor is attached. In order to carry out the rough correction of the inclination, a manual tilt stage is installed between the ceramics workpiece and the table of the polishing machine. Figure 6(a) shows the configuration of the tilt stage and the measuring workpiece. Figure 6(b) shows a schematic diagram of the inclination angle compensation method by using the tilt stage. As shown in Fig. 6(b), the distance between the displacement sensor and the workpiece surface at the both ends of the scanning line is measured, and then the inclination angle is adjusted by the tilt stage so that the deviation of the distance measurement results are less than $2 \mu \mathrm{m}$. As a result, the tilt error caused by the mechanical inclination can be reduced.

The tilt error which cannot be removed by the tilt stage is compensated by the mathematical processing based on the least square method and the rotation matrix. Figure 7 shows the schematic of the measurement error caused by the 
tilt of the workpiece surface. As shown in Fig. 7, the measurement result in $Y$ direction can be expressed as follows.

$$
Y_{\text {real }}=\frac{y}{\cos \theta}
$$

where, $y$ is the ideal distance between the laser displacement sensor and the measuring surface, $\theta$ is the declination angle of the workpiece surface. Therefore, the error of the measurement in the $Y$ direction can be expressed as follows.

$$
Y_{\text {error }}=\frac{y}{\cos \theta}-y
$$

In order to realize the sub-micrometric accuracy, $Y_{\text {error }}$ has to be less than $0.1 \mu \mathrm{m}$. According to Eq. (3), $y$ has to be satisfied the following inequality.

$$
\begin{aligned}
& \frac{y}{\cos \theta}-y \leq 0.1 \\
& \theta \leq \cos ^{-1}\left(\frac{10 y}{1+10 y}\right)
\end{aligned}
$$

Furthermore, the difference between the displacement at $X_{0}$ and that at $X_{1}$ can be expressed as follows.

$$
d=x \tan \theta
$$

where, $x$ is the displacement of the displacement of the linear stage of the polishing machine in $X$ direction.

According to Eqs. (5) and (6), the inequality about the difference of the displacement can be obtained from the following inequality .

$$
d \leq x \tan \left[\cos ^{-1}\left(\frac{10 y}{1+10 y}\right)\right]
$$

Then, the linearity of the laser displacement sensor also affects the result of the form measurement. As shown in Table 2, the linearity of the sensor is $\pm 0.05 \%$ of FS. Therefore, the error of the linearity can be expressed as follow:

$$
Y_{\text {error }}= \pm 5 \times 10^{-4} \times d
$$

To achieve the sub-micrometric accuracy of the form profile measurement, $Y_{\text {error }}$ has to be less than $0.1 \mu \mathrm{m}$. According to Eq. (8), $d$ has to be satisfied the following inequality.

$$
d \leq \pm \frac{0.1}{5 \times 10^{-4}}= \pm 200 \mu \mathrm{m}
$$

In order to realize the sub-micrometric measuring accuracy, the parameter $d$ has to be satisfied the inequalities (7) and (9). By using the manual tilt stage, the declination of the workpiece can be corrected with micrometric order. However, it is difficult for the manual tilt stage to correct the inclination of the workpiece with sub-micrometric accuracy. Therefore, the compensation by coordinate transformation has been employed. Figure 9 shows a schematic of angle compensation with data processing. The least square method and rotation matrix are used for the compensation with the data processing. The gradient of the least square fitted curve can be expressed as follow.

$$
a=\frac{n \sum_{i} x_{i} y_{i}-\sum_{i} x_{i} \sum_{i} y_{i}}{n \sum_{i} x_{i}^{2}-\left(\sum_{i} x_{i}\right)^{2}}
$$

where $a$ is the gradient of the least square fitted curve, $n$ is the number of the sampling, $x_{i}$ and $y_{i}$ are the coordinate data of the measuring points. The relation between the gradient $a$ and the angle $\theta$ can be expressed as follows.

$$
\theta=\tan ^{-1} a
$$

By using the estimated $\theta$, the rotational matrix which rotational center is the first measuring point can be obtained from the following matrix

$$
\left(\begin{array}{l}
x^{\prime} \\
y^{\prime}
\end{array}\right)=\left(\begin{array}{cc}
\cos \theta & \sin \theta \\
-\sin \theta & \cos \theta
\end{array}\right)\left(\begin{array}{l}
x \\
y
\end{array}\right)
$$

where $x$ and $y$ are the coordinates before the rotation, and $x^{\prime}$ and $y^{\prime}$ are the after the rotation respectively. After the mechanical compensation with the tilting stage, the compensation by using the coordinate transformation with data process is carried out. By utilizing two types of the compensation method, the errors which are occurred by the inclination will be possible to be eliminated. 
Ito, Matsuura, Meguro, Goto, Shimizu, Gao, Adachi and Omiya,

Journal of Advanced Mechanical Design, Systems, and Manufacturing, Vol.8, No.4 (2014)

Table 3 Reflectivity of the measurement samples

\begin{tabular}{c|c|c}
\hline \hline & $\begin{array}{c}\text { Silicon nitride } \\
(R a=0.01 \mu \mathrm{m})\end{array}$ & $\begin{array}{c}\text { Metallic gauge block } \\
(R a=0.01 \mu \mathrm{m})\end{array}$ \\
\hline Incident light intensity & $597.3 \mu \mathrm{W}$ & $597.7 \mu \mathrm{W}$ \\
\hline Reflected light intensity & $45.9 \mu \mathrm{W}$ & $304.0 \mu \mathrm{W}$ \\
\hline Reflectivity & $8.8 \%$ & $50.8 \%$ \\
\hline \hline
\end{tabular}

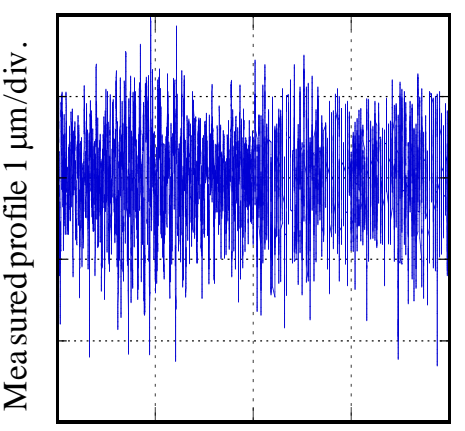

Position in $X$ direction $5 \mathrm{~mm} /$ div.

(a) Silicon nitride

$(R a=0.42 \mu \mathrm{m})$

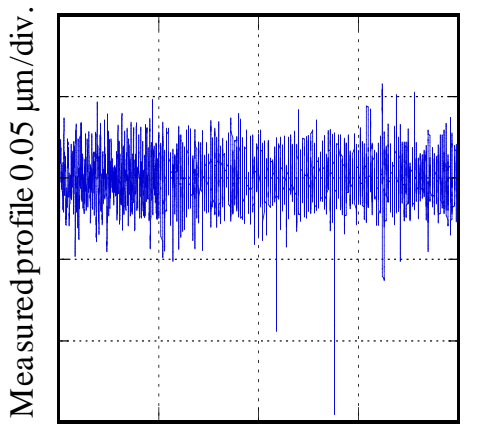

Position in $X$ direction $5 \mathrm{~mm} /$ div.

(b) Metallic gauge block $(R a=0.01 \mu \mathrm{m})$

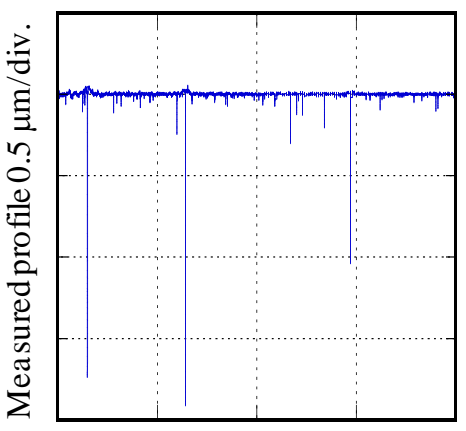

Position in $X$ direction $5 \mathrm{~mm} / \mathrm{div}$

(c) Silicon nitride $(R a=0.01 \mu \mathrm{m})$

Fig. 8 Surface roughness measurements by using a surface form measurement machine

\subsection{Stability error}

Stability of the constructed measurement system was investigated. In this study, two kinds of silicon nitride samples and a metallic gauge block were used as the measuring sample to compare the difference of the reflectivity. Two types of the silicon nitride samples were processed in different surface roughness. The surface roughness of those samples were measured by a surface form measuring machine (Form Talysurf, PGI 420) and the arithmetical mean roughness of three samples were estimated. Figure 8 shows the measurement result of surface roughness by using the surface form measurement machine. As shown in Fig. 8, the arithmetical mean roughness $(\mathrm{Ra})$ of the two silicon nitride samples were $0.42 \mu \mathrm{m}$ and $0.01 \mu \mathrm{m}$, respectively. Similarly, the arithmetical mean roughness of the metallic gauge block was $0.01 \mu \mathrm{m}$. The triangulation method laser displacement sensor, which is used for the measurement of the surface form profile, can measure the displacement by detecting the change of the spot position of the laser beam reflected on the measuring sample surface. Therefore, it is difficult for the material with low reflectivity to measure the distance precisely. Table 3 shows the refractivity of the measurement samples used in this study. The reflectivity shown in Table 3 was calculated based on the intensity difference between the incident light and reflected light of the triangulation method laser displacement sensor, which was measured by the optical power meter. As shown in table 3, the reflectivity of the silicon nitride sample is lower that of the metallic gauge block significantly, so the reflected light intensity that is detected by the CMOS sensor is also reduced. In order to investigate the influence of the reflectivity of the measurement samples, the stability evaluation of the laser displacement sensor output was carried out by changing the measurement sample. The measurement samples was fixed on the table of the polishing machine, and then the distance between the displacement sensor and the measurement sample was set to $8 \mathrm{~mm}$, which was a reference distance of the used laser displacement sensor. The linear stages of the polishing machine were kept stationary to monitor the behavior of the sensor output. The sampling frequency of the displacement sensor was set to be $100 \mathrm{~Hz}$. 


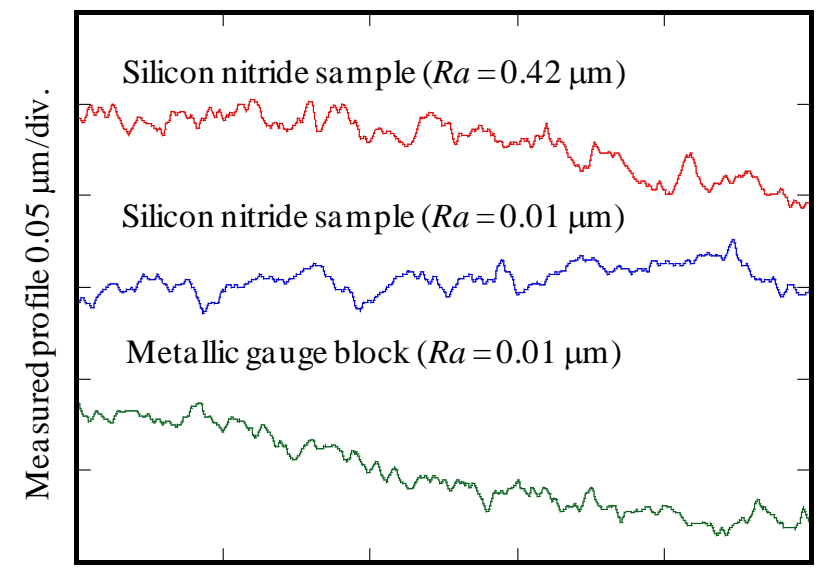

Time 50 sec./div.

Fig. 9 Experimental result of the stability evaluation

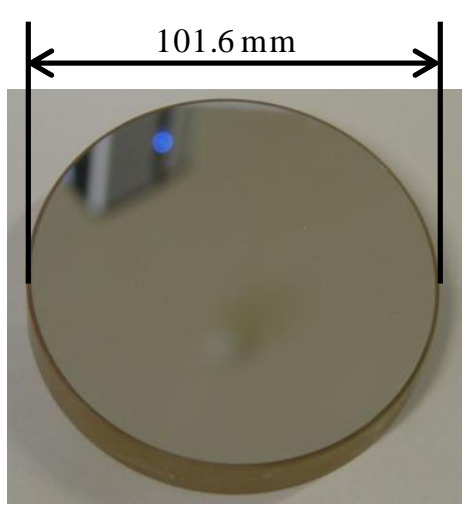

(a) A flat mirror

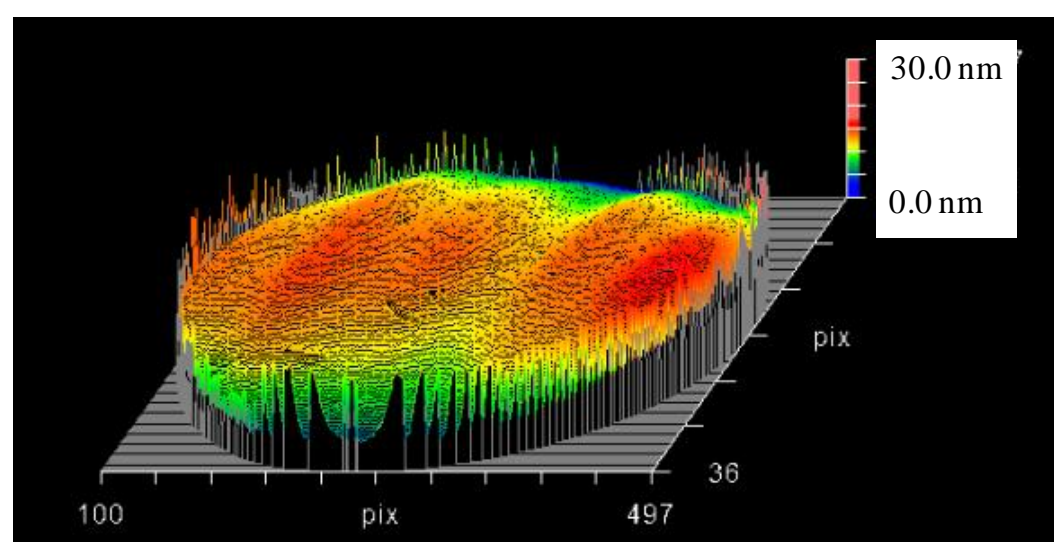

(b) Measurement result by a laser interferometer

Fig. 10 A flat mirror used as a reference sample

The displacement signal of the sensor was output through a digital low-pass filter with the cutoff frequency of $0.1 \mathrm{~Hz}$. Figure 9 shows the experimental result of the stability evaluations of three types of the samples on the measurement system. As shown in Fig. 9, the sensor outputs were stable within a range of $0.1 \mu \mathrm{m}$. The difference in the stability owing to the surface roughness and the reflectivity was not observed. Furthermore, it could be confirmed that the difference in the stability due to the surface roughness was very small. Therefore, the stability of the constructed measurement system is enough high for the sub-micrometric surface form profile measurement. In addition, the stability of the measurement system is hardly influenced by the surface roughness and the reflectivity of the measuring object.

\subsection{Motion error}

In the constructed on-machine measurement system, the laser displacement sensor is scanned on the sample surface by the linear guide of the polishing machine. Therefore, the measurement accuracy is also influenced by the motion accuracy of the linear stages. A motion error of the measurement system was evaluated by using a flat mirror. Figure 10 shows a flat mirror that is employed as a reference sample to evaluate the motion error of the measurement system. The profile irregularity of the flat mirror was measured by a laser interferometer (Verifire XPZ, Zygo). Figure 10(b) shows the measurement result by the laser interferometer. As shown in this figure, the profile irregularity of the flat mirror used in this study was $30 \mathrm{~nm}$. The laser displacement sensor was moved in $X$ direction at $0.08 \mathrm{~mm} / \mathrm{s}$ by the linear stage of the polishing machine. The sampling frequency and the cutoff frequency of the digital low-pass filter were the same 


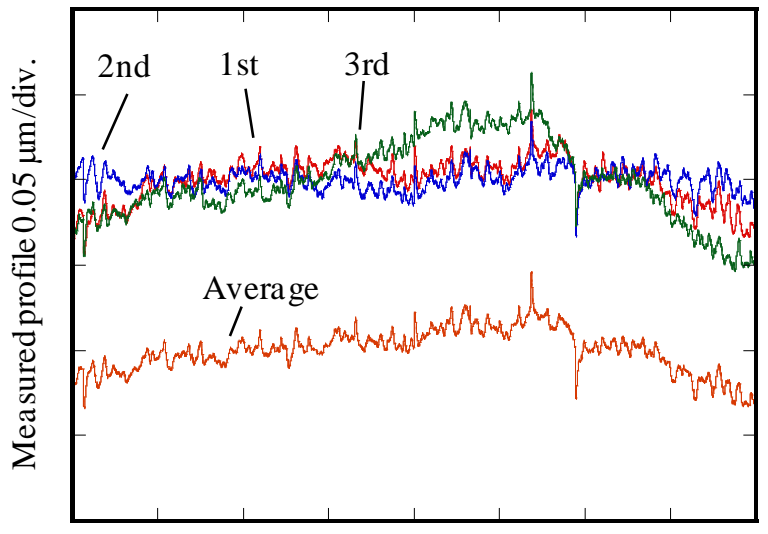

Position in $X$ direction $10 \mathrm{~mm} / \mathrm{div}$.

Fig. 11 Experimental result of the motion accuracy evaluations

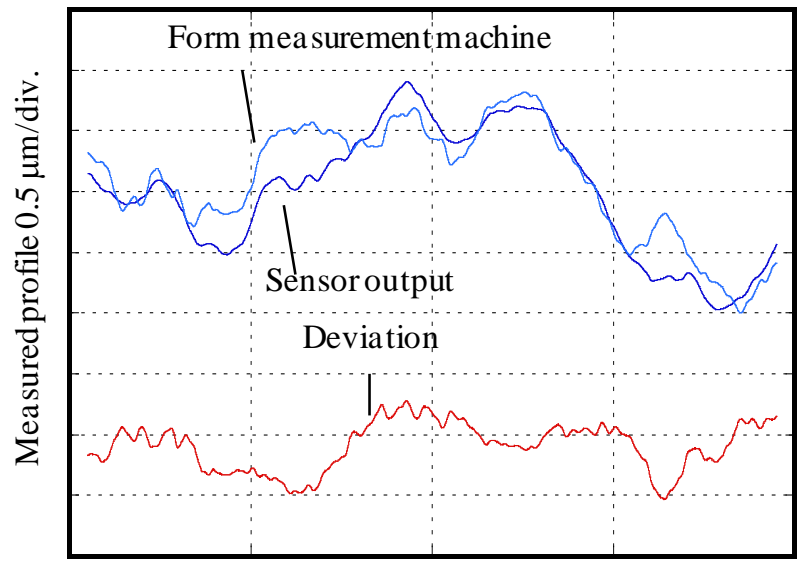

Position in $X$ direction $5 \mathrm{~mm} / \mathrm{div}$.

Fig. 12 Experimental result of the form profile measurement of a silicon nitride sample $(R a=0.42 \mu \mathrm{m})$

as the stability evaluation. The measurement was carried out three times and calculated the average of the three measurement results. Figure 11 shows the experimental result of the motion accuracy evaluations. The surface of the flat mirror was scanned by the laser displacement sensor. The vertical axis of Fig. 11 indicates the measured profile that is calculated based on the output of the displacement sensor. As a result, the motion error was $0.16 \mu \mathrm{m}$. As can be seen table 1, the straightness of linear slide of the polishing machine in $X$ direction is $0.2 \mu \mathrm{m}$ and it is almost the same to the motion error. Therefore, it was estimated that the major factor of the motion error would be induced by straightness of the linear stages of the machining tool. Since the straightness is the unique parameter of the polishing machine, it is possible to compensate the straightness error of the linear guide.

\section{On-machine measurement of the ceramics sample}

The surface form profiles of the samples shown in table 3 were measured on the polishing machine by using the developed measurement system. Those samples were fixed on the tilt stage which is mounted to the table of the polishing machine in turn. The distance between the laser displacement sensor and the surface of the sample was set to be $8 \mathrm{~mm}$, and then the rough correction of the inclination was carried out by using the tilt stage. The surface profile of the workpieces was measured by scanning the laser displacement sensor. The laser displacement sensor was moved in $X$ direction at $0.08 \mu \mathrm{m} / \mathrm{s}$ by the linear stage of the polishing machine. The sampling frequency of the displacement sensor was set to be $100 \mathrm{~Hz}$. The output signal of the displacement sensor was recorded through the low-pass filter with a cutoff frequency of $0.1 \mathrm{~Hz}$. Figure 12 shows the result of the form profile measurement of the silicon nitride sample of which $R a$ is $0.42 \mu \mathrm{m}$. The same ceramics sample was also measured by the surface form measurement machine. The 


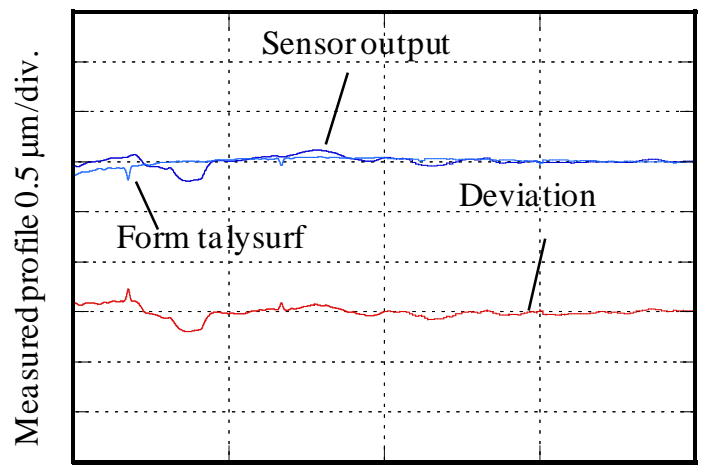

Position in $X$ direction $5 \mathrm{~mm} / \mathrm{div}$.

(a) Silicon nitride sample $(R a=0.01 \mu \mathrm{m})$

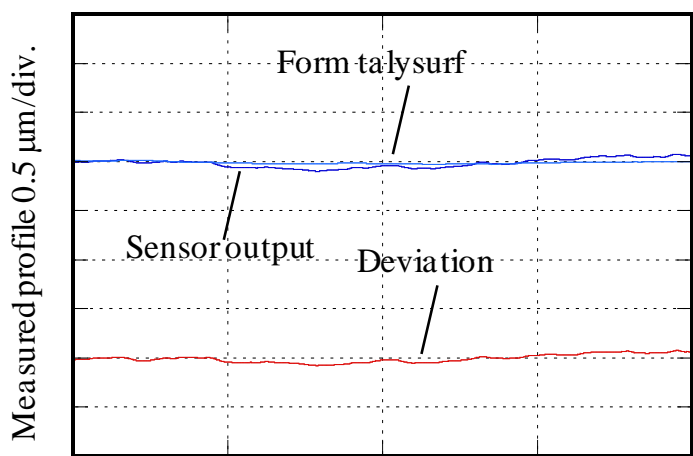

Position in $X$ direction $5 \mathrm{~mm} /$ div.

(b) Metallic gauge block

$(R a=0.01 \mu \mathrm{m})$

Fig. 13 Experimental result of the form profile measurement of a silicon nitride sample and a metallic gauge block

inclination of the measurement result that could not be removed by the mechanical correction of the tilt stage was mathematically corrected by Eq. (12). The maximum deviation shown in Fig. 12 indicates the difference between the measurement result of the laser displacement sensor and that of the surface form measurement machine. As shown in Fig. 12, the deviation was $0.81 \mu \mathrm{m}$. In this measurement, the surface form profile measured by the laser displacement sensor did not completely agree with the measurement result obtained by the surface form profile measurement machine because the sample had to be attached to the different measurement instrumentations. However, it could be confirmed that the deviation between two measurement instruments was sub-micrometric. Figure 13(a) shows the result of the form profile measurement of the silicon nitride sample of which $R a$ is $0.01 \mu \mathrm{m}$. As well as Fig. 12, the deviation of two measurement instruments was estimated. As shown in Fig. 13, the deviation was $0.42 \mu \mathrm{m}$. The difference of both results of the silicon nitride samples were within $1.0 \mu \mathrm{m}$. Those results indicate that the measuring accuracy of the developed on-machine measurement system is equivalent of that of the surface form measurement machine and it can realize the precision measurement of the surface profile with sub-micrometric accuracy. Furthermore, figure 13(b) shows the result of the form profile measurement of a metallic gauge block of which $R a$ is $0.01 \mu \mathrm{m}$. As shown in Fig. 13(b), the deviation of two measurement instruments was $0.15 \mu \mathrm{m}$. In comparison with the measurement result of the silicon nitride samples, the order of the deviation was almost same. Consequently, it can be confirmed that the measurement system using the laser displacement sensor can obtain the surface profile independently of the reflectivity of the workpiece material.

\section{Conclusion}

An on-machine measurement system by using a laser displacement sensor has been developed for the surface profile measurement of the ceramics workpieces. Since the laser displacement sensor is enough small to be mounted on the tool post of the polishing machine, the surface profile of the measuring samples can be measured on the table of the machining tool.

The measurement system could realize the measurement range of several hundred millimeters with sub-micrometric straightness by utilizing the linear stages of the ultra-precision polishing machine. Therefore, the laser displacement sensor allowed the continuously measurement of the ceramics surface with nanometric resolution in noncontact condition. The laser displacement sensor can measure the surface profile regardless of the reflectivity and the surface roughness of the measuring samples. The output of the laser displacement sensor was enough stable for the measurement with sub-micrometric accuracy. The stability was within $0.1 \mu \mathrm{m}$, and the motion accuracy was $0.16 \mu \mathrm{m}$ respectively. Consequently, the stability and the motion error were enough for the sub-micrometric form profile measurement. The surface form of the ceramics samples were measured by the constructed measurement system. The results of the surface profile obtained by the constructed measurement system were compared with the results of the surface form measurement machine. The measurement results show that the deviation between the result of the 
constructed system and the surface measuring machine are within $1.0 \mu \mathrm{m}$. From these results, the measuring accuracy of the proposed system was equivalent of that of a surface from measuring machine regardless of the difference of the surface roughness.

\section{References}

Goto, S., Shimizu, Y., Lee, J.C., Ito, S., Gao, W., Adachi, S., Omiya, K., Sato, H., Hisada, Y., and Kubota, H., On-Machine Profile Measurement of Large Mirror for Satellite (1st Report): - Construction of the Measurement System and Development of the Alignment Methods -, Journal of the Japan Society for Precision Engineering, Vol. 78 (2012) pp. 631-635.

Kuwata, T., Morita, N., Watanabe, T. and Yoshida, Y., Study on the Laser processing of the Covalent Bond-Ceramics (4th Report) -Analysis of the Deep Hole Processing-, Journal of the Japan Society for Precision Engineering, Vol. 59, No. 11 (1993), pp. 1797-1802.

Moriwaki, T., Shamoto, E. and Inoue, K., Ultraprecision Ductile Cutting of Glass by Applying Ultrasonic Vibration, Annals of the CIRP, Vol. 4, No. 1 (1992), pp. 141-144.

Mou, J. and Richard, C.L., A method for enhancing the accuracy of CNC machine tools for on-machine inspection, Journal of Manufacturing Systems, Vol. 11 (1992) pp. 229-237.

Nakamoto, K., Linear Motor Drive Ultra Precision Machine using Ceramics made Aerostatic Guideway, Journal of the Japan Society for Precision Engineering, Vol. 72, No. 4 (2006) pp. 427-430.

Onaka, T. and Nakagawa, T., A 3.5 m space infrared telescope for mid- and far-infrared astronomy, Advances in Space Research, Vol. 36, No. 6 (2005) pp. 1123-1130.

Otsuka, J., Ultraprecision Positioning, Journal of the Japan Society for Precision Engineering, Vol.75, No.1 (2009) pp. 82-84.

Rahman, M.S., Saleh, T., Lim, H.S., Son, S.M. and Rahman, M., Development of an on-machine profile measurement system in ELID grinding for machining aspheric surface with software compensation, International Journal of Machine Tools and Manufacture, Vol. 48, No. 7-8 (2008) pp. 887-895.

Sato, K and Komatsuzaki, T, Research of the technology of the grinding process for fine ceramics, Report of the research of the Ibaraki industrial technology center, Vol. 21 (1992) pp. 115-119.

Shimizu, Y., Goto, S., Lee, J.C., Ito, S., Gao, W., Adachi, S., Omiya, K., Sato, H., Hisada, T., Saito, Y. and Kubota, H., Fabrication of large-size $\mathrm{SiC}$ mirror with precision aspheric profile for artificial satellite, Precision Engineering, Vol. 37 (2013) pp. 640-649.

Sugawara, J., Low Thermal Expansion Advanced Ceramics for Precision Engineering, Journal of the Japan Society for Precision Engineering, Vol. 79, No. 4 (2013) pp. 82-84.

Suzuki, H., Hamada, S., Okino, T., Kondo, M., Yamagata, Y. and Higuchi, T., Ultraprecision finishing of micro-aspheric surface by ultrasonic two-axis vibration assisted polishing, Annals of the CIRP, Vol. 4, No. 1 (1992), pp. 141-144.

Ueno, S., On Machine Measuring System for Machine tool, Journal of the Japan Society for Precision Engineering, Vol. 75, No. 11 (2009) pp.1269-1272. 